\title{
Trial-two goal arm alternation to direction of movement in trial-one straight alley
}

MICHAEL F. SHERRICK AND WILLIAM N. DEMBER

UNIVERSITY OF CINCINNATI

The previously reported, unexpected finding that rats may alternate on trial 2 in the goal arms of a T-maze the direction of their movement in the starting-stem of a trial-1 T-maze was followed up in the present study with a minor procedural variation: trial-1 movement occurred in a simple straight alley. Once again, there was a tendency for trial-2 T-maze goal-arm choice to take the rat in a direction opposite to that enforced by the orientation of the trial-1 alley.

In a thorough investigation of the stimuli which might serve as cues for spontaneous alternation, Douglas (1964) concluded that rats alternate to only one intramaze and one extra-maze cue. The odor trail left by $S$ on the maze floor during its first trial in the T-maze is the only effective intra-maze cue. The single extramaze cue is directional in nature: Salternates its prior direction of movement.

According to Douglas, these two alternation cues can be investigated in isolation. Odor trail cues are eliminated by changing paper flooring in the mazes between trials. To neutralize the direction of movement cue, the goal arms of the trial-1 and trial-2 T-maze are set perpendicular to each other.

Although such orthogonal maze alignment precludes goal arm alternation, Dember, Sherrick, \& Harris (1966) suggest that this procedure may endow significance to the orientation of the trial-1 starting stem. In two experiments a tendency was exhibited for $S$ to enter the trial-2 goal arm which was opposite in direction to its enforced direction of movement in the trial-1 starting stem. Both the novelty of this finding and certain weaknesses in the data on which it was based dictated further investigation.

The present experiment was designed to explore further the proposed effect of trial-1 starting stem on trial-2 goal arm choice, but with a procedural simplification introduced, i.e., the use of a straight alley instead of a $\mathrm{T}$-maze on trial 1 . In this way, potential confounding effects of trial-1 goal arm movement are eliminated, thus allowing maximal expression of the tendency-should it indeed be present.

\section{Method}

The Ss were 48 gentled, male, hooded rats of the Long-Evans strain, approximately 130-135 days old. The Ss had run previously in a separate but related alternation experiment; otherwise they were experimentally naive.

Apparatus consisted of a straight alley and a $\mathrm{T}$-maze. The straight alley was $30 \mathrm{in}$. long, 4-1/2 in. wide, and 6 in. high, with a starting box 6-1/2 in. in length. The
T-maze dimensions were as follows: all alleys 4 in. wide and 6 in. high, $18 \mathrm{in}$. goal arms and starting stem, and a 6 in. starting box. Both straight alley and T-maze interiors were painted gray. Paper flooring was changed after each trial to eliminate any possible odor trail effects from one rat to another.

The experimental conditions were achieved by varying the orientations of both the trial-1 straight alley and trial-2 T-maze, yielding the elght conditions shown in Fig. 1. Each $S$ was run once in each of the eight conditions in a Latin square design.

\section{Results}

Data are presented for only those 38 of the 48 Ss that actually $\mathrm{ran}$ in the straight alley and the T-maze in at least four of the eight experimental conditions. Conclusions based on these data, however, do not differ from those based on the unselected data from all 48 Ss.

In Table 1, frequency of trial-2 arm choice and percentage alternation are reported for each of the eight conditions. Note that the slight variation in $\mathrm{N}$ is related to the failure of some rats to run in each of the conditions.

Several statistical analyses were employed, all of which convincingly indicate the predicted effect of trial-1 straight alley orientation on trial-2 goal arm choice. The effect was present in seven of the eight

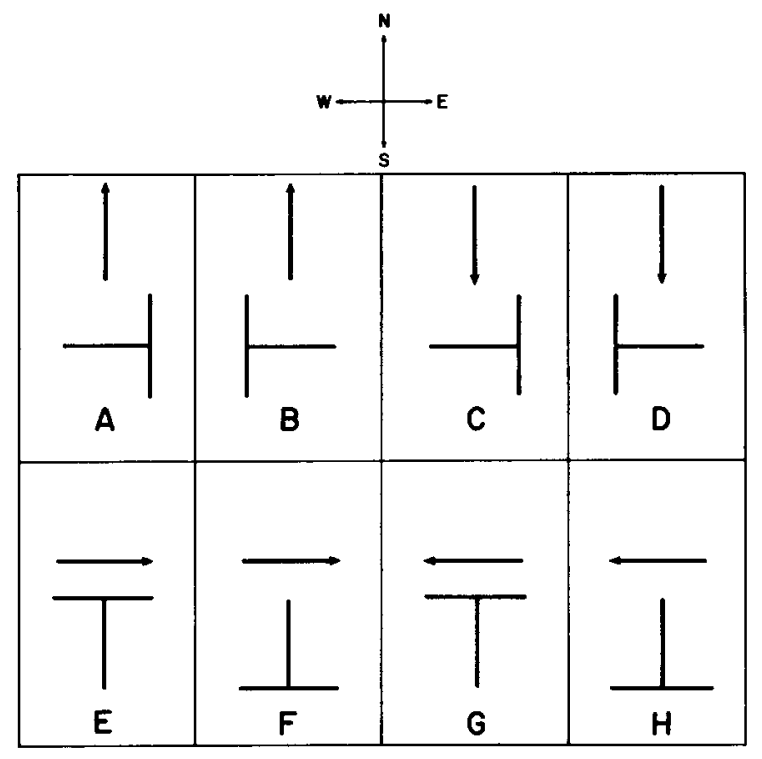

Fig. 1. The eight experimental conditions. Arrows indicate the direction of movement in the trial-1 straight alley. 
Table 1. Trial-Two Goal Arm Choice and Alternation Percentage in Each of the Eight Conditions

\begin{tabular}{lrrrrrrrrrr} 
Condition & & A & B & C & D & & E & F & G & H \\
\hline \multirow{2}{*}{ Direction of Choice } & South & 23 & 27 & 13 & 18 & West & 17 & 21 & 11 & 12 \\
& North & 10 & 8 & 20 & 15 & Eost & 15 & 10 & 24 & 20 \\
Percent Alternation & & 70 & 77 & 61 & 45 & & 53 & 68 & 69 & 62 \\
\hline
\end{tabular}

conditions. With data from the eight conditions combined, there were 167 alternations to 97 repetitions. This $63 \%$ rate of alternation was significantly greater than the $50 \%$ expected by chance $\left(X^{2}=18.6, p<.01\right)$.

Since the experiment involved repeated measures, one additional analysis was made. Each $S$ was assigned a score corresponding to the total number of times it alternated; the mean alternation score, over $38 \mathrm{Ss}$, was compared with the hypothetical chance mean via a t-test, and again the difference was highly significant $(t=5.15$, $\mathrm{p}<.01)$.

\section{Discussion}

The data confirm the prediction that trial-2 goal arm choice will reflect the tendency to move in a direction opposite to that enforced by straight alley orientation on trial 1. These results support the attribution by Dember, Sherrick, \& Harris (1966) of potential significance to the grientation of the trial-1, T-maze starting stem.

While extending the generality of the direction of movement cue, as discovered by Douglas (1964), the present results, along with those reported by Dember, Sherrick, \& Harris (1966), suggest that it may be difficult to investigate alternation behavior in the absence of this cue. Apparently, the procedure developed by Douglas (i.e., the perpendicular alignment of $\mathrm{T}$-mazes on trials 1 and 2) does not completely eliminate direction of movement as an alternation cue. This poses a problem for the investigation of any other possible alternation cue in isolation.

\section{References}

Dember, w. N., Sherrick, M. F., \& Harris, R. P. Jr. Trial-two goal arm alternation to orientation of trial-one starting stem. Psychon. Sci., 1966, 6, 31-32.

Douglas, R. J. An analysis of spontaneous alternation cues. Unpublished doctoral dissertation, University of Michigan, 1964. 